\title{
Transmisión intergeneracional del castigo físico en la niñez
}

Recibido: 18 de diciembre de 2018 - Aceptado: 30 de noviembre de 2020

Doi: https://doi.org/10.12804/revistas.urosario.edu.co/economia/a.7519

\author{
Marisa Bucheli* \\ Máximo Rossi**
}

\section{Resumen}

El uso de la violencia física moderada en la crianza de niños es una práctica extendida y aceptada como una medida disciplinaria. Sin embargo, hay pruebas de que ejercer estas prácticas durante la infancia produce efectos negativos en el curso de la vida adulta. En ese sentido, el principal objetivo de esta investigación es comprobar si existe asociación entre el haber recibido castigo físico en la infancia como instrumento disciplinario y, la transmisión intergeneracional de esta conducta con los hijos. Para ello, se utilizó la Encuesta de Situaciones Familiares (ESF) realizada en el 2007, financiada por la Agencia Nacional de Investigación e Innovación de Uruguay (ANII) y el Fondo de las Naciones Unidas para la Infancia (Unicef).

El análisis de los datos estadísticos se realizó mediante un modelo probit con variables dependientes para determinar la utilización del castigo físico como instrumento disciplinario, la frecuencia con la que la persona recibió castigo en la niñez y, variables — binarias e independientes- de género, edad y nivel educativo. Así mismo, se tuvieron en cuenta

* Departamento de Economía, Facultad de Ciencias Sociales, Universidad de la República, Uruguay. Email: marisa.bucheli@cienciassociales.edu.uy orcid: https://orcid. org/0000-0003-0159-4712

* Departamento de Economía, Facultad de Ciencias Sociales, Universidad de la República, Montevideo. Email: maximo.rossi@cienciassociales.edu.uy orcID: https:// orcid.org/0000-0002-4116-922X

Para citar este artículo: Bucheli, M., \& Rossi, M. (2021). Transmisión intergeneracional del castigo físico en la niñez. Revista de Economía del Rosario, 24(1), 5-19. https://doi. org/10.12804/revistas.urosario.edu.co/economia/a.7519 
las opiniones y actitudes positivas frente al caso "golpear como una técnica disciplinaria" y su inferencia en la implementación de esta acción en el futuro.

Por último, los resultados para el caso uruguayo evidenciaron que existe transmisión intergeneracional en el uso del castigo físico como técnica disciplinaria tanto para hombres como mujeres, sin diferencia de género y, que la opinión sobre el uso del castigo físico produce cambios en los resultados de la trasmisión intergeneracional.

Palabras clave: castigo; hogar; niños; violencia.

Clasificación JEL: J12, J13, D0.

\title{
Intergenerational Transmission of Physical Punishment in Childhood
}

\begin{abstract}
The use of moderate physical violence in raising children is a widespread and accepted practice as a disciplinary measure. However, there is evidence that these practices during childhood have negative effects on the course of adult life, which motivated the analysis of the intergenerational transmission of this behavior. The Family Situations Survey carried out in 2007, financed by the National Agency for Research and Innovation of Uruguay (anii) and the United Nations Fund for Children (unicef), was used.

Statistical data analysis was performed using a probit model with dependent variables to determine the use of physical punishment as a disciplinary instrument, the frequency with which the person received punishment in childhood, and variables - binary and independent - of gender, age and educational level. Likewise, the positive opinions and attitudes were taken into account regarding the case "hitting as a disciplinary technique" and their inference in the implementation of this action in the future.
\end{abstract}

Finally, for the Uruguayan case, we found evidence that there is intergenerational transmission of the use of physical punishment as a disciplinary technique. The use of corporal punishment by parents is related to its approval, both in men and in women, without statistically significant differences. On the other hand, when introducing the opinion on the use of this technique, the intergenerational transmission of the use of physical punishment in the case of women is maintained, but it loses significance in the case of men.

Keywords: violence; home; children; beaten; punishments.

\section{Transmissão intergeracional do castigo físico na infância}

\section{Resumo}

O uso de violência física moderada na criação dos filhos é uma prática amplamente difundida e aceita como medida disciplinar. No entanto, há evidências de que essas práticas na infância têm efeitos negativos no curso da vida adulta. Isso motiva a análise da transmissão intergeracional desse comportamento. Utilizou-se o Levantamento de 
Situações Familiares realizado em 2007, financiado pela Agência Nacional de Pesquisa e Inovação do Uruguai (anii) e pelo Fundo das Nações Unidas para a Infância (unicef).

A análise estatística dos dados foi realizada por meio de um modelo probit com variáveis dependentes para determinar o uso do castigo físico como instrumento disciplinar, a frequência com que a pessoa recebia castigo na infância e as variáveis - binárias e independentes - de sexo, idade e nível educacional. Da mesma forma, foram tidas em consideração as opiniões e atitudes positivas relativamente ao caso "bater como técnica disciplinar" e a sua inferência na futura implementação desta ação.

Finalmente, para o caso uruguaio, encontramos evidências de que há transmissão intergeracional do uso do castigo físico como técnica disciplinar. O uso de castigos físicos por parte dos pais está relacionado à sua aprovação, tanto por homens quanto por mulheres, sem diferenças estatisticamente significativas. Por outro lado, ao introduzir a opinião sobre o uso dessa técnica, a transmissão intergeracional do uso do castigo físico no caso das mulheres é mantida, mas perde significância estatística no caso dos homens.

Palavras-chave: violência, casa, crianças, espancados, punições.

Classificação JEL: J12, J13, D0.

\section{Introducción}

El uso del castigo corporal como técnica de disciplina infantil ha sido considerado tradicionalmente como legítimo. Sin embargo, existe una gran cantidad de evidencia empírica — para los países desarrollados-, que señala al castigo físico durante la infancia como predictor de una serie de resultados negativos durante la vida adulta — ansiedad y conductas agresivas - (Ateah \& Durrant, 2005; Cuartas et al., 2020; Pace et al., 2019). De igual manera, hay estudios adicionales sobre la implementación del castigo físico, que como técnica correctiva, se transmite de padres a hijos (Bandura, 1969, 1973; Gage \& Silvestre, 2010; Tajima, 2000, 2002; Graziano et al., 1996; Rodríguez \& Sutherland, 1999; Socolar \& Stein, 1995). Aunque en estudios recientes ya no se evidencia el castigo físico como técnica disciplinaria o correctiva, lo cual se interpreta como cambio cultural en la sociedad (Ateah \& Durrant, 2005).

En ese marco, este trabajo hace un aporte a la literatura sobre el tema enfocado en los países en vía de desarrollo, en específico, de Uruguay. El objetivo es analizar la transmisión intergeneracional del uso de castigo físico como técnica disciplinaria en la crianza de los niños uruguayos. Además, se estudia la asociación entre el haber experimentado castigo físico en la niñez $\mathrm{y}$, la opinión que se tiene al respecto con el uso de este instrumento en la crianza de sus hijos. La información se recopiló de mujeres y hombres que conviven con hijos menores de 22 años, en los cuales, el $30 \%$ de las madres 
—que están en una relación-y el $17 \%$ de los padres, declaran usar el castigo físico con sus hijos cuando tienen un mal comportamiento o no obedecen.

Para identificar la transmisión intergeneracional del uso de estas técnicas disciplinarias, se realizó una Encuesta de Situaciones Familiares (ESF) en la cual se preguntó por la frecuencia con que los niños recibían castigo físico cuando sus padres lo consideraban pertinente y, si el adulto experimentó castigo corporal durante su infancia como método correctivo.

Así pues, el texto se estructura de la siguiente manera: primero, se hace una revisión de la literatura sobre el tema; luego una presentación de los datos y la metodología usada; más adelante, se muestran los resultados; y por último, las conclusiones y alcances de la investigación.

\section{Revisión de la literatura}

La mayoría de trabajos investigativos sobre este tema han sido realizados en países desarrollados, en los cuales se encuentra una relación positiva entre el castigo físico experimentado en la niñez y el uso de este con sus propios hijos (Gage \& Silvestre, 2010; Tajima, 2002, 2000; Rodríguez \& Sutherland, 1999; Graziano et al., 1996; Socolar \& Stein, 1995). De acuerdo con la teoría del aprendizaje social, los comportamientos agresivos se transmiten por aprendizaje o por imitación. En términos generales, esta teoría sostiene que los individuos incorporan a su vida el comportamiento de aquellos con quienes se identifican, por tanto, las personas que fueron castigadas en la niñez tienen una probabilidad mayor de usar el castigo físico durante la vida adulta (Bandura, 1969, 1973). De esta manera, las actitudes y opiniones de los individuos sobre el uso del castigo físico, pueden funcionar como mediadoras entre la experiencia de castigo temprano y la aplicación de castigo corporal a los hijos.

Dicho lo anterior, las actitudes como mediadoras han sido estudiadas por diversos autores. Bower y Knutson (1996), muestran que los individuos disciplinados con el uso del castigo físico, son más propensos a evaluar positivamente esta técnica disciplinaria y, por lo tanto, a utilizarla con sus hijos.

Otras investigaciones de Ateah y Durrant (2005), Graziano et al. (1996) y Cappa y Khan (2011), encontraron que las actitudes de los padres son los mejores predictores en el uso del castigo físico como técnica disciplinaria.

Respecto a las diferencias entre padres y madres, se ha detectado que el efecto de recibir castigo en la niñez sobre las prácticas de castigo físico con hijos, es mayor para las madres que para los padres, y en ciertos casos, el efecto para los padres desaparece (Lukenheimer et al., 2006). Varios análisis encontraron que las mujeres son más propensas que los hombres a usar la 
violencia física para disciplinar a los niños, lo que estaría relacionado con que están más tiempo con sus hijos en el hogar (Jackson et al., 1999). Aunque en un estudio sobre el comportamiento de madres, Ateah y Durrant (2005) no hallan evidencia de transmisión intergeneracional en el castigo físico infantil, mencionan que este hallazgo podría deberse a razones espurias, como la dificultad de recordar experiencias pasadas o a la renuencia de informar las experiencias de violencia. No obstante, se puede argumentar que existieron cambios en el comportamiento que podrían estar interrumpiendo la transmisión intergeneracional en el uso del castigo físico en los niños. Este cambio se basaría en variaciones del marco legal y las campañas públicas de concientización para modificar las actitudes hacia el uso de esa técnica disciplinaria.

Del mismo modo, existen otros factores que pueden impulsar o restringir el uso del castigo físico en la niñez como las características de los padres, los niños, el entorno social, entre otros. Por otra parte, la mayoría de la evidencia indica que las personas de mayor edad son menos propensas a apoyar el castigo físico y a ejercer violencia (Tajima, 2002; Douglas, 2006).

Por último, la conexión de la relación entre el nivel educativo y el uso del castigo físico, no es sólida. Tajima (2002) informa sobre varios estudios que encontraron el castigo físico más probable para el nivel educativo más alto. Aunque otras investigaciones concluyen que los padres de bajo nivel educativo son más propensos al uso del castigo físico (Cappa \& Khan, 2011; Jackson et al., 1999), mientras que Buntain-Ricklefs et al. (1994), no observaron una relación significativa entre estas variables. Desde otra perspectiva, Erten y Keskin (2019) hallaron un impacto positivo de los programas de educación compulsiva en la reducción del castigo físico como elemento disciplinario para el caso de Turquía.

\section{Datos y metodología}

Para desarrollar esta investigación se utilizó la Encuesta de Situaciones Familiares (ESF) realizada en el 2007 por la Universidad de la República, financiada por la Agencia Nacional de Investigación e Innovación de Uruguay (ANII) y el Fondo de las Naciones Unidas para la Infancia (Unicef). El objetivo principal de la ESF era recopilar información sobre el matrimonio, el divorcio, la fecundidad, la crianza de los hijos y el bienestar infantil. La muestra abarcó a 1.224 mujeres entre 25 y 62 años que viven en el área metropolitana de Montevideo, a través de entrevistas presenciales. Y solo fueron consultadas sobre la utilización de distintas prácticas disciplinarias, mujeres con niños menores de 22 años de edad. 
Ahora bien, cuando las mujeres convivían con un hombre en casamiento o unión libre, este también era entrevistado, por lo tanto, se recogió su uso de prácticas disciplinarias. Además, a ambos se les preguntó con qué frecuencia recibían castigo físico como técnica disciplinaria en la niñez. Ya que el interés de la investigación era comparar hombres y mujeres, se trabajó con la submuestra de quienes viven en pareja. Como resultado, la base de datos contiene información de 481 padres y 481 madres, pero debido a valores perdidos se utilizaron 415 observaciones de cada tipo. En el resto de este documento, las mujeres y hombres son denominados como madres y padres casados, aunque incluyen personas en unión libre y lazos no biológicos.

\section{Variables}

La pregunta sobre si el individuo utiliza el castigo físico como técnica disciplinaría con su hijo, se incluye en un bloque que indaga diferentes formas de castigo. A los individuos se les pregunta: “Cuándo sus hijos se portan mal o no obedecen después de decirles que se detengan muchas veces, ¿con qué frecuencia...?". De este modo, fueron investigados ocho posibles comportamientos, que no corresponden con el orden de aparición a una graduación de conductas bajo ninguna norma social: los pone en penitencia, les grita, les pega, les prohíbe hacer algo que les gusta, se retira del lugar donde están, los echa de la casa, no suele enojarse con sus hijos o deja de hablarles.

Como proxy del castigo físico se usó la información reportada por el comportamiento que se menciona en tercer lugar: "pegar". Las respuestas precodificadas son "nunca", "a veces" y "con frecuencia". Se empleó esta información para construir la variable dependiente ("castigo físico") que toma valor 1 cuando la respuesta es "a veces" o "frecuentemente" y 0 cuando contesta "nunca".

Dicho lo anterior, las variables cuyo potencial relacionamiento con el uso del castigo físico en la niñez que interesan a esta investigación son:

- Recibir en la niñez castigo físico como técnica disciplinaria. En la encuesta se preguntó "¿Con qué frecuencia le pegaban a usted cuando era niño para corregirle?". Las posibles respuestas eran: "nunca", "rara vez", "con frecuencia", "casi siempre" y "siempre". Se agregaron las frecuencias "casi siempre" y "siempre", y se construyó una variable con valores de 1 a 4 , que aumenta con la regularidad que la persona ha recibido castigo.

- Opinión sobre el uso del castigo físico como técnica disciplinaria. En la encuesta se preguntó sobre el acuerdo o desacuerdo de esta práctica, con una serie de declaraciones sobre actitudes y opiniones hacia la maternidad, la 
violencia y el matrimonio, entre otros temas. Las respuestas precodificadas eran: "muy de acuerdo", "de acuerdo", "ni de acuerdo ni en desacuerdo", "de acuerdo" o "muy en desacuerdo". También se usó la opinión sobre la afirmación "A los niños hay que pegarles para corregir su conducta" y se agregaron las respuestas correspondientes a "muy de acuerdo" y "de acuerdo" para crear una variable que toma los valores 1 a 4 , que aumenta con el grado aprobación frente al uso de la técnica disciplinaria.

- Otros controles. Se tuvo en cuenta la edad y educación de la persona. La edad es una variable binaria que toma el valor 1 para individuos menores de 40 años y 0 en el otro caso. La educación se identificó con tres variables binarias: primaria completa, educación secundaria —completa o incompleta-y educación terciaria — completa o incompleta-.

En la tabla 1 se presentan los valores promedio de las variables utilizadas en el estudio. Los hombres declaran pegarle menos (18\%) a sus hijos que las mujeres (30\%). Este resultado resalta la importancia del problema y es consistente con la correspondencia que se ha obtenido en la mayoría de las investigaciones sobre otros países. Page et al. (2019) analizando 72 países de bajo y mediano ingreso, encontraron que el $43 \%$ de los niños fueron golpeados durante el mes anterior a la entrevista; Runyan et al. (2010) con una muestra de mujeres de 19 comunidades de Brasil, Chile, Egipto, India, Filipinas y Estados Unidos, rastrearon que entre un $16 \%$ y $76 \%$ de las madres han reportado que ellas o sus maridos han golpeado a los niños en el año pasado a la entrevista.

Vale la pena resaltar que la base de datos de este trabajo no ofrece una información amplia sobre el marco social y el entorno familiar de los entrevistados, lo cual potencialmente podría estar afectando la conducta y opinión de los individuos (Bisin \& Verdier, 2011).

Tabla 1. Valor medio

\begin{tabular}{lcc}
\hline & Mujeres & Hombres \\
\hline Recibió castigo físico en la niñez & 1.924 & 2.001 \\
& $(0.050)$ & $(0.043)$ \\
Castigo físico como técnica disciplinaria & 1.943 & 1.948 \\
Menor de 40 años & $(0.041)$ & $(0.038)$ \\
& 0.527 & $0.392^{* * *}$ \\
& $(0.026)$ & $(0.027)$
\end{tabular}




\begin{tabular}{lcc}
\hline & Mujeres & Hombres \\
\hline Educación primaria & 0.190 & 0.193 \\
Educación secundaria & $(0.021)$ & $(0.021)$ \\
& 0.548 & 0.584 \\
Educación terciaria & $(0.026)$ & $(0.025)$ \\
& 0.262 & $0.223^{*}$ \\
Variable dependiente: castigo físico & $(0.023)$ & $(0.021)$ \\
Número de casos & 0.297 & $0.185^{* * *}$ \\
\hline
\end{tabular}

Nota: (error estándar entre paréntesis). ${ }^{* *} \mathrm{p}<0.01,{ }^{* *} \mathrm{p}<0.05,{ }^{*} \mathrm{p}<0.1$ en la prueba de diferencia de medias entre hombres y mujeres.

Fuente: elaboración propia.

\section{Estrategia empírica}

Como se ha mencionado, el primer objetivo era analizar la asociación entre haber recibido castigo físico en la niñez con el uso del castigo físico como técnica disciplinaria con sus hijos. Para esto, se estimó la probabilidad de ejercer castigo físico sobre los hijos utilizando un modelo probit:

$$
P(Y=1 / X)=\Phi\left(b_{0}+b_{1} h+0_{2} E+b_{3} N+b_{4} P+b_{5} E h+b_{6} N h+b_{7} P h\right)
$$

En la ecuación (1), la letra $Y$ toma valor 1 cuando el individuo utiliza castigo físico y 0 cuando no lo utiliza. Para la estimación de la probabilidad, el modelo probit utiliza la función de distribución acumulada en la norma estándar que se representa con la letra $\Phi$. Las variables de las que depende la probabilidad se denotan $h, E, N$ y $P$. La letra $h$ corresponde a una variable binaria que toma valor 1 para los hombres y 0 para las mujeres; $E$ es una variable binaria que toma valor 1 para los menores de 40 años y 0 para los mayores; $N$ es un vector de variables binarias que distingue el nivel educativo; y $P$ es la variable que recoge el pasado del individuo - frecuencia con que recibió castigo físico en la niñez-. Además de introducir cada una de estas variables, también se consideró la interacción de $h$ con $E, N$ y $P$, lo que admite que la relación de cada variable con $Y$ pueda ser diferente para hombres y mujeres. En particular, el coeficiente $b_{4}$ recoge la relación entre ser mujer y el uso del castigo físico, mientras que el coeficiente $b_{7}$ toma la diferencia entre hombres y mujeres en relación a dicho uso. 
Como se ha comentado, el principal interés del estudio es la estimación del efecto marginal de la variable "haber recibido castigo físico en la niñez", la cual se calcula como derivada de la expresión (1) con respecto a $P$. El efecto marginal indica cuánto cambia la probabilidad —en puntos porcentualesde usar el castigo físico en sus hijos cuando aumenta la frecuencia de haber recibido castigo físico en la infancia. Aunque la estimación no implica relaciones causales, un efecto positivo significativo puede interpretarse como un soporte de la presencia de transmisión generacional. Así mismo, como se ha incluido la interacción entre $P$ y $h$ como variable explicativa, es posible calcular el efecto para hombres y mujeres por separado y, someter a prueba la hipótesis de que la diferencia de género es nula. En especial, se presenta el efecto promedio.

El segundo objetivo es explorar hasta qué punto las opiniones y actitudes afectan el uso del castigo físico. Para hacer esto, se estimó un modelo probit como el anterior, agregándosele la opinión sobre el uso del castigo físico como técnica disciplinaria. Formalmente, usando la letra $O$ para representar las variables de opiniones, se consideró:

$$
\begin{gathered}
P(Y=1 / X)=\Phi\left(b_{0}+b_{1} h+b_{2} E+b_{3} N+b_{4} P+b_{5} E h+b_{6} N h+b_{7} P h+\right. \\
\left.b_{8} O+b_{7} P h\right)
\end{gathered}
$$

En la anterior estimación, el principal interés es el efecto marginal de la variable que refleja haber recibido castigo físico y, la variable de opinión sobre su uso. Es decir, las derivadas de $Y$ con respecto a $P$ y con respecto a 0 , que se calculan nuevamente para hombres y mujeres por separado. Se pudo interpretar que una disminución del efecto de la variable "recibió castigo físico en la niñez" articulado con un efecto positivo de la variable "castigo físico como técnica disciplinaria", significa que el efecto de la experiencia de castigo opera a través de la formación de opiniones.

\section{Resultados}

En el apéndice al final de este documento, se presentan los resultados de las estimaciones de las ecuaciones (1) y (2). A continuación se analiza la existencia de transmisión intergeneracional y el papel de las actitudes sobre el uso del castigo físico como técnica disciplinaria a través de sus efectos marginales y probabilidades predichas. 


\section{Evidencia sobre la transmisión intergeneracional del uso de castigo físico}

En la tabla 2 se presenta la estimación del efecto marginal de la variable que recopila la frecuencia con que se recibió castigo físico para corregir el comportamiento durante la niñez. El efecto indica que, en promedio, la probabilidad de utilizar el castigo físico aumenta 5.2 puntos porcentuales para los hombres y 9.2 para las mujeres.

A pesar de que la diferencia de género es 4 puntos porcentuales, las pruebas de contraste indican que no hay diferencias significativas.

También se reporta la probabilidad predicha de ejercer castigo físico para las distintas respuestas brindadas sobre la niñez. Para el caso de los hombres, la probabilidad crece de 0.12 cuando contesta no haber sido nunca castigado, a 0.27 cuando dice haber sido corregido siempre o casi siempre a través del uso de castigo físico. Para las mujeres, la probabilidad predicha crece de 0.16 a 0.38 .

En suma, hay evidencia de que existe transmisión intergeneracional del uso de castigo físico como técnica disciplinaria tanto para hombres como mujeres, sin diferencia de género estadísticamente significativa en los niveles de confianza habitualmente utilizados.

Tabla 2. Relación entre el uso de castigo físico y haber recibido castigo físico en la niñez con base en la estimación de un modelo probit

\begin{tabular}{lccc}
\hline \multicolumn{1}{c}{ Variable dependiente: uso de castigo } & Hombres & Mujeres & Diferencia \\
\hline Efecto marginal promedio de haber recibido castigo & $0.0517^{* *}$ & $0.0922^{* * *}$ & -0.0405 \\
Probabilidad predicha cuando se recibió castigo: & & & \\
Nunca & $0.1178^{* * *}$ & $0.1635^{* * *}$ & -0.0457 \\
Rara vez & $0.2024^{* * *}$ & $0.3579^{* * *}$ & -0.1556 \\
Con frecuencia & $0.2345^{* * *}$ & $0.4263^{* * *}$ & -0.1918 \\
Casi siempre/Siempre & $0.2712^{* * *}$ & $0.3843^{* * *}$ & -0.1131 \\
\hline
\end{tabular}

Nota: ${ }^{* * *} \mathrm{p}<0.01,{ }^{* *} \mathrm{p}<0.05,{ }^{*} \mathrm{p}<0.1$.

Fuente: elaboración propia.

\section{Opiniones sobre las técnicas disciplinarias como un canal} de transmisión intergeneracional de la violencia

Al introducir esta opinión se producen cambios en los resultados de la trasmisión intergeneracional (tabla 3). Es decir, no se recoge evidencia sobre la existencia de transmisión intergeneracional para los hombres, lo que 
sugiere que el efecto reflejado en la especificación anterior está mediado por la opinión. El efecto marginal promedio de la opinión es aproximadamente 12 puntos porcentuales para los hombres y 15 puntos porcentuales para las mujeres, pero la diferencia no es estadísticamente significativa.

Tabla 3. Relación entre el uso del castigo físico recibido en la niñez como técnica disciplinaria y la opinión sobre su uso con base a la estimación de un modelo probit

\begin{tabular}{lccc}
\hline & Hombres & Mujeres & Diferencia \\
\hline Efecto marginal promedio de haber recibido castigo & 0.0247 & $0.0809^{* * *}$ & $-0.0562^{*}$ \\
Probabilidad predicha cuando se recibió castigo: & & & \\
Nunca & $0.1543^{* * *}$ & $0.1823^{* * *}$ & -0.0281 \\
Rara vez & $0.1960^{* * *}$ & $0.3457^{* * *}$ & $-0.1497^{* * *}$ \\
Con frecuencia & $0.1973^{* * *}$ & $0.4047^{* * *}$ & $-0.2074^{* * *}$ \\
Casi siempre/Siempre & $0.2396^{* * *}$ & $0.3874^{* * *}$ & -0.1478 \\
Efecto marginal promedio de opinión & $0.1244^{* * *}$ & $0.1466^{* * *}$ & -0.0222 \\
Probabilidad predicha según opinión: & & & \\
Nunca & $0.0820^{* * *}$ & $0.1202^{* * *}$ & -0.0382 \\
Rara vez & $0.1595^{* * *}$ & $0.3249^{* * *}$ & $-0.1655^{* * *}$ \\
Con frecuencia & $0.4868^{* * *}$ & $0.5509^{* * *}$ & -0.0642 \\
Casi siempre/Siempre & $0.4062^{* * *}$ & $0.4859^{* * *}$ & -0.0797 \\
\hline
\end{tabular}

Nota: ${ }^{* *} \mathrm{p}<0.01,{ }^{* *} \mathrm{p}<0.05,{ }^{*} \mathrm{p}<0.1$.

Fuente: elaboración propia.

En el caso de las mujeres, el efecto marginal de recibir castigo en la niñez como técnica disciplinaria se reduce de 9.2 puntos porcentuales en la especificación anterior a 8.1 en la nueva, pero sigue siendo significativo. A su vez, el efecto marginal de la opinión es 15 puntos porcentuales. Ello indica que, para las mujeres, si bien la opinión también opera como un mediador de la trasmisión intergeneracional, no es el único mecanismo que la explica.

\section{Limitaciones}

Este trabajo tuvo una serie de limitaciones para tener en cuenta al momento de considerar sus resultados. En primer lugar, es necesario reiterar que el trabajo no encuentra encadenamientos causales, sino una correlación estadísticamente significativa entre las variables planteadas. 
En segundo lugar, al trabajar con información autorreportada, se podría estar sesgando los resultados obtenidos. Por ejemplo, las personas más educadas podrían desinformar respecto al uso del castigo como recurso disciplinario, en la medida que reconocen los efectos negativos sobre el niño.

En tercer lugar, el autorreporte cuando está referido a instancias lejanas en el tiempo puede presentar sesgos asociados a la memoria sobre esos hechos. Además, el marco cultural actual podría estar afectando la visión y recuerdo de las experiencias pasadas.

En cuarto lugar, la base de datos no da información sobre otros aspectos del entorno familiar y social que, potencialmente podrían estar afectando la conducta y opinión de los individuos.

\section{Conclusiones}

El uso del castigo físico en la crianza de los hijos es una práctica que tradicionalmente ha sido aceptada como una técnica disciplinaria. No obstante, es probable que nuevos enfoques en la legislación y las campañas educativas llevadas a cabo en distintos países, pongan barreras cada vez mayores a la continuidad de estas prácticas de generación en generación. La falta de consenso en los países desarrollados y la escasa evidencia para países en vía de desarrollo, motiva a realizar el estudio de la potencial transmisión intergeneracional del uso de castigo físico para el caso uruguayo.

En este estudio se encontró evidencia de que existe transmisión intergeneracional del uso de castigo físico como técnica disciplinaria tanto para mujeres como para hombres, sin diferencias de género estadísticamente significativas. También, se demostró que la opinión es un mediador de esta transmisión, más fuerte en el caso de los hombres que de las mujeres.

Por último, estos resultados lucen interesantes para el diseño de políticas específicas contra la reducción del uso de castigo físico en los niños. En efecto, más allá de que otras variables no observadas que afectan esta conducta — como el descontrol—, el castigo está respaldado por una visión positiva de la utilidad del instrumento que podría ser modificada a través de programas públicos de educación e información.

\section{Referencias}

Ateah, C., \& Durrant, J. E. (2005). Maternal use of physical punishment in response to child misbehavior: Implications for child abuse prevention. Child Abuse \& Neglect, 29(2), 169-185. https://doi.org/10.1016/j.chiabu.2004.10.010 
Bandura, A. (1969). Principles of behavior modification. Holt, Rinehart, and Winston, Inc.

Bandura, A. (1973). Aggression: A social learning analysis. Prentice Hall.

Bisin, A., \& Verdier, T. (2011). The economics of cultural transmission and socialization. In J. Benhabib, A. Bisin \& M. Jackson (Eds.), Handbook of social economics (pp. 339-416). North-Holland.

Bower, M., \& Knutson, J. (1996). Attitudes toward physical discipline as a function of disciplinary history and self-labeling a physically abuse. Child Abuse E Neglect, 20(8), 689-699. https://doi.org/10.1016/01452134(96)00057-9

Buntain-Ricklefs, J., Kemper, K., Bell, M., \& Babonis, T. (1994). Punishment: what predicts adult approval. Child Abuse \& Neglect, 18(11), 945-955. https://doi.org/10.1016/S0145-2134(05)80005-5

Cappa, C., \& Khan, S. M. (2011). Understanding caregivers' attitudes towards physical punishment of children: Evidence from 34 low-and middleincome countries. Child Abuse E Neglect, 35(12), 1009-1021. https://doi. org/10.1016/j.chiabu.2011.10.003

Cuartas, J., Ward, K., Ma, J., \& Grogan-Kaylor, A. (2020). Physical punishment and Colombian children and adolescents' cognitive and behavioral outcomes. Journal of Applied Developmental Psychology, 68, 101-140. https:// doi.org/10.1016/j.appdev.2020.101140

Douglas, E. (2006). Family violence socialization in childhood and later life approval of corporal punishment: A cross-cultural perspective. American Journal of Orthopsychiatry, 16(1), 23-30. https://doi.org/10.1037/00029432.76.1.23

Erten, B., \& Keskin, P. (2019). Breaking the cycle? Education and the intergenerational transmission of violence. Review of Economics and Statistics, 102(2), 252-268. https://doi.org/10.1162/rest_a_00824

Gage, A. J., \& Silvestre, E. A. (2010). Maternal violence victimization, and child physical punishment in Peru. Child Abuse \& Neglect, 34(7), 522533. https://doi.org/10.1016/j.chiabu.2009.12.004

Graziano, A., Hambler, J., \& Plante, W. (1996). Subabusive violence in child rearing in middle-class American families. Pediatrics, 98(4), 845-848. https://pediatrics.aappublications.org/content/98/4/845

Jackson, S., Thompson, R., Christiansen, E., Colman, R., Wyatt, J., Buckendahl, C., Wilcox, B., \& Peterson, R. (1999). Predicting abuse-prone parental attitudes and discipline practices in a nationally representative sample. Child Abuse E Neglect, 23(1), 15-29. https://doi.org/10.1016/S01452134(98)00108-2 
Lunkeiheimer, E., Kittler, J., Olson, S., \& Kleinberg, F. (2006). The intergenerational transmission of physical punishment: differing mechanism in mothers' and fathers' endorsement? Journal Family violence, 21(8), 509-519. https://doi.org/10.1007/s10896-006-9050-2

Pace, G. T., Lee, S. J., \& Grogan-Kaylor, A. (2019). Spanking and young children's socioemotional development in low-and middle-income countries. Child Abuse \& Neglect, 88, 84-95. https://doi.org/10.1016/j. chiabu.2018.11.003

Rodríguez, C., \& Sutherland, D. (1999). Predictors of parents' physical disciplinary practices. Child Abuse $\mathcal{E}$ Neglect, 23(7), 651-657. https://doi. org/10.1016/S0145-2134(99)00043-5

Runyan, D. K., Shankar, V., Hassan, F., Hunter, W. M., Jain, D., Paula, C. S., Bangdiwala, S., Ramiro, L., Muñoz, S., Vizcarra, B., \& Bordin, I. A. (2010). International variations in harsh child discipline. Pediatrics, 126(3), 701711. https://doi.org/10.1542/peds.2008-2374

Socolar, R. R., \& Stein, R. E. (1995). Spanking of infants and toddlers: Maternal belief and practice. Pediatrics, 95(1), 105-111. https://pediatrics. aappublications.org/content/95/1/105

Tajima, E. A. (2000). The relative importance of wife abuse as a risk factor for violence against children. Child Abuse $\mathcal{E}$ Neglect, 24(11), 1383-1398. https://doi.org/10.1016/S0145-2134(00)00194-0

Tajima, E. A. (2002). Risk factors for violence against children: comparing homes with and without abuse. Journal of Interpersonal Violence, 17(2), 122-149. https://doi.org/10.1177/0886260502017002002

\section{Apéndice}

Tabla 4. Estimación de los modelos probit. Variable dependiente: castigo físico

\begin{tabular}{|c|c|c|}
\hline & \multicolumn{2}{|c|}{ Mujeres casadas } \\
\hline & (1) & (2) \\
\hline \multirow{2}{*}{ Recibió castigo físico en la niñez (mujer) } & $0.282^{* * *}$ & $0.265^{* * *}$ \\
\hline & $(0.0713)$ & $(0.0735)$ \\
\hline \multirow{2}{*}{ Castigo físico como técnica disciplinaria (mujer) } &.-- & $0.481^{* * *}$ \\
\hline &.-- & $(0.0944)$ \\
\hline \multirow{2}{*}{ Menores de 40 años (mujer) } & $0.336^{* *}$ & $0.253^{*}$ \\
\hline & $(0.138)$ & $(0.142)$ \\
\hline
\end{tabular}




\begin{tabular}{|c|c|c|}
\hline \multirow{2}{*}{ Educación secundaria (mujer) } & 0.181 & 0.158 \\
\hline & $(0.202)$ & $(0.205)$ \\
\hline \multirow{4}{*}{ Educación terciaria (mujer) } & -0.0389 & -0.0827 \\
\hline & $(0.226)$ & $(0.229)$ \\
\hline & \multicolumn{2}{|c|}{ Mujeres casadas } \\
\hline & (1) & (2) \\
\hline \multirow{2}{*}{ Hombre } & -0.00344 & -0.0385 \\
\hline & $(0.383)$ & $(0.484)$ \\
\hline \multirow{2}{*}{ Recibió castigo físico en la niñez (hombre) } & -0.0835 & -0.163 \\
\hline & $(0.123)$ & $(0.130)$ \\
\hline \multirow{2}{*}{ Castigo físico como técnica disciplinaria (hombre) } &.-- & 0.0353 \\
\hline & $-\cdot^{-}$ & $(0.148)$ \\
\hline \multirow{2}{*}{ Menores de 40 años (hombre) } & -0.0827 & 0.0264 \\
\hline & $(0.197)$ & $(0.209)$ \\
\hline \multirow{2}{*}{ Educación secundaria (hombre) } & -0.203 & -0.158 \\
\hline & $(0.305)$ & $(0.308)$ \\
\hline \multirow{2}{*}{ Educación terciaria (hombre) } & -0.0832 & -0.00680 \\
\hline & $(0.315)$ & $(0.330)$ \\
\hline \multirow{2}{*}{ Constante } & $-1.369^{* * *}$ & $-2.239^{* * *}$ \\
\hline & $(0.250)$ & $(0.312)$ \\
\hline Número de casos & 822 & 822 \\
\hline
\end{tabular}

Nota: Error estándar entre paréntesis. ${ }^{* *} \mathrm{p}<0.01,{ }^{* *} \mathrm{p}<0.05,{ }^{*} \mathrm{p}<0.1$.

Fuente: elaboración propia. 